\section{Fatores de risco associados ao nascimento de recém-nascidos de muito baixo peso em uma população de baixa renda}

\author{
Risk factors associated with very low birth weight \\ in a low-income population
}

\author{
${ }^{1}$ Hospital Geral de Caxias do \\ Sul, Universidade de Caxias \\ do Sul, Caxias do Sul, Brasil. \\ 2 Faculdade de Saúde \\ Pública, Universidade de \\ São Paulo, São Paulo, Brasil. \\ Correspondência \\ B. F. Araújo \\ Hospital Geral de Caxias \\ do Sul, Universidade de \\ Caxias do Sul. \\ Rua Orestes Baldisserotto \\ 931, Caxias do Sul, RS \\ 95032-260, Brasil. \\ bfaraujo@terra.com.br
}

\section{Abstract}

This study aimed to identify risk factors associated with very low birth weight in a general hospital in Caxias do Sul, Rio Grande do Sul State, Brazil. This was a case-control study of 200 newborns with birth weight from 500 to 1,499g (cases) and 400 with birth weight from 3,000 to $3,999 \mathrm{~g}$ (controls). Infants were from singleton pregnancies, and their mother had received prenatal care at public health services. The dependent variable was birth weight, and independent variables included socioeconomic status, schooling, and gestational and birth status. Univariate and multivariate analyses were performed with a 5\% level of significance. Mortality in very low birth weight newborns was $32.5 \%$. The limits of viability were $600 \mathrm{~g}$ for birth weight and 26 weeks for gestational age. Variables related to very low birth weight were: maternal age $>35$ years $(p=0.01)$, lack of prenatal care $(p<0.0001)$, illness during the index pregnancy ( $p=0.03)$, maternal hypertension ( $p=0.007)$, hospitalization during pregnancy $(p<0.0001)$, and prior history of low birth weight $(p<0.0001)$. Many premature births were due to avertable factors.

Low Birth Weight Infant; Premature Infant; Poverty
Breno Fauth de Araújo 1

Ana Cristina d'Andretta Tanaka 2

\section{Introdução}

Nas últimas décadas, o Brasil vem passando pelo processo de transição epidemiológica, com modificação nos padrões de morbidade e mortalidade, com uma progressiva redução nas taxas de mortalidade e fecundidade acompanhada pelo aumento das doenças degenerativas, da obesidade e das doenças causadas pelo homem, fenômeno este característico dos países desenvolvidos 1 . Nesse período, as taxas de mortalidade infantil foram reduzidas pela metade no país e, em algumas regiões, a um quarto dos valores iniciais. Essa queda ocorreu inicialmente no componente pós-neonatal e no final da década de 90 houve a inversão esperada, com a mortalidade neonatal passando a ser quase duas vezes maior que a pósneonatal.

Os principais determinantes do risco de morrer no período neonatal são o baixo peso de nascimento e a idade gestacional menor do que 37 semanas.

Nos países onde as taxas de mortalidade infantil são baixas e existe um alto desenvolvimento tecnológico, como nos Estados Unidos e Canadá, a queda da mortalidade nos últimos anos tem ocorrido basicamente entre os recémnascidos de peso de nascimento menor do que $1.000 \mathrm{~g}$. A sobrevida dos recém-nascidos com peso entre 500 e 599g aumentou de zero, em 1980, para quase $80 \%$ em 1993, mostrando o extraordinário progresso alcançado na diminuição da 
mortalidade destes recém-nascidos com extremo baixo peso 2 .

Entretanto, a melhor forma de se baixar a taxa de mortalidade e diminuir os problemas e seqüelas relacionados com a prematuridade ainda é pela diminuição dos nascimentos prematuros, o que contribui significativamente para a diminuição da mortalidade infantil, por meio da redução do número de casos em risco de morrer.

É interessante notar, contudo, que a fre qüência de partos prematuros e de recém-nascidos de muito baixo peso vem crescendo em todo o mundo. De 1981 para 1998, o número de partos prematuros aumentou nos Estados Unidos $23 \%$, estando atualmente em $11,6 \%$, e a taxa de recém-nascidos de muito baixo peso aumentou $24 \%$, passando de $1,15 \%$ para $1,43 \%$ dos nascimentos ${ }^{3}$.

Caxias do Sul apresentou, em 2003, uma freqüência de 1,6\% de recém-nascidos de muito baixo peso, sendo estes recém-nascidos os responsáveis por $42 \%$ de todos os óbitos infantis da cidade.

Kramer et al. 4 descreveram como causas desse aumento de nascimentos de muito baixo peso a elevação da proporção de mães idosas, as más condições sócio-econômicas, o comportamento de risco durante a gestação e os novos métodos de fertilização, gerando nascimentos múltiplos, com o conseqüente aumento dos partos prematuros.

Além do aumento das gestações múltiplas relacionadas com os tratamentos de infertilidade, que nos países em desenvolvimento, como o Brasil, são responsáveis somente por um pequeno número de nascimentos prematuros, contribuem, também, para esses nascimentos as doenças maternas pré-gestacionais, a préeclâmpsia, as infecções gênito-urinárias, o baixo nível educacional da gestante, o desemprego, o hábito de fumar e o uso de drogas na gestação, a ausência de pré-natal, o parto prematuro anterior, entre outras 3 .

Dessa forma, o presente estudo tem como objetivo identificar os fatores de risco associados ao nascimento de recém-nascidos de muito baixo peso em uma população de baixa renda da Região Sul do Brasil.

\section{Material e método}

É um estudo caso-controle de base hospitalar, cujos casos e controles nasceram entre março de 1998 e dezembro de 2004 no Hospital Geral de Caxias do Sul, Rio Grande do Sul, Brasil, que é um hospital que atende exclusivamente pacientes do Sistema Único de Saúde (SUS).
A população do estudo foi constituída por 600 recém-nascidos, sendo 200 no grupo casos e 400 no grupo controles. O grupo de casos foi constituído por todos os recém-nascidos com peso de nascimento entre 500 e $1.499 \mathrm{~g}$ e o grupo de controles por recém-nascidos com peso de nascimento entre 3.000 e $3.999 \mathrm{~g}$, nascidos no mesmo dia do caso. Foram selecionados, por sorteio, dois controles para cada caso identificado.

No estudo foram incluídos os recém-nascidos de gestação única, cujas mães não realizaram o pré-natal ou o fizeram, exclusivamente, na rede pública de saúde e que durante o período gestacional e de parto residiram no Município de Caxias do Sul. Foram excluídos os recém-nascidos com peso ao nascer entre 1.500 e $2.999 \mathrm{~g}$ e os que apresentaram peso igual ou maior do que 4.000g.

A amostra foi estimada baseando-se em erro tipo I (alfa) máximo de 5\%, um poder estatístico mínimo de $80 \%$ e uma razão caso:controle de 1:2. Levando-se em conta uma exposição entre os controles de $5 \%$ e uma OR estimada de 3,0, o cálculo da amostra foi de 141 casos e 282 controles, sendo este número acrescido em $40 \%$ para possibilitar o ajuste dos fatores de confusão e potencial interação em modelos de regressão múltipla, resultando em uma amostra de 197 casos e 395 controles.

Desse modo, considerando os aspectos estatísticos e logísticos envolvidos optou-se por selecionar 200 casos e 400 controles.

As informações deste estudo foram obtidas nos prontuários das gestantes e dos recém-nascidos e digitadas em um banco de dados do programa Epi Info 6.0 (Centers for Disease Control and Prevention, Atlanta, Estados Unidos), sendo a análise das informações realizada no programa SPSS versão 12.0 (SPSS Inc., Chicago, Estados Unidos).

Os dados foram coletados dos prontuários da gestante e do recém-nascido. Alguns dados relacionados com a gestante, como a renda per capita e outras variáveis, foram obtidos de protocolos de pesquisa realizados no serviço de obstetrícia do hospital. Em relação às variáveis dos recém-nascidos de muito baixo peso internados na UTI neonatal, todos os dados vêm sendo sistematicamente coletados ao longo dos anos, no momento da alta hospitalar e digitados em um banco de dados, garantindo, assim, a confiabilidade da informação.

A variável dependente foi o peso ao nascer do recém-nascido e as variáveis independentes foram agrupadas em variáveis maternas e variáveis relacionadas à gestação e ao parto. Para verificar a presença ou não de associação entre as variáveis independentes e o desfecho 
estudado estimou-se a odds ratio (OR) bruta e ajustada para cada associação de interesse. $\mathrm{Na}$ análise univariada foi aplicado o teste $\mathrm{t}$ de Student para as variáveis contínuas e o teste quiquadrado $\left(\chi^{2}\right)$ para as variáveis dicotômicas. A análise multivariada, visando à identificação de possíveis vieses de confusão, foi realizada por meio da regressão logística não-condicional, pelo método Enter.

Os critérios de seleção das variáveis para o modelo de regressão logística envolveram a significância estatística $(\mathrm{p}<0,05)$ na análise univariada e/ou a relevância clínico-epidemiológica da variável mediante o conhecimento vigente.

O estudo foi aprovado pelo Comitê de Ensino e Pesquisa do Hospital Geral de Caxias do Sul e pelo Comitê de Ética em Pesquisa da Faculdade de Saúde Pública da Universidade de São Paulo.

\section{Resultados}

Os recém-nascidos do grupo controle apresentaram uma média de peso ao nascer de $3.374 \mathrm{~g}$ $(\mathrm{DP}=259 \mathrm{~g})$ e uma idade gestacional média de $39,2$ semanas ( $D P=1,1)$, com a menor idade gestacional encontrada de 35 semanas e a maior de 42 semanas. Sete recém-nascidos dessa população $(1,8 \%)$ tiveram idade gestacional menor do que 37 semanas.

O tipo de parto predominante foi o normal (77,5\%). A mediana do Apgar no $1^{\circ}$ minuto foi de 9 e somente $7 \%$ dos recém-nascidos deste grupo necessitaram de algum tipo de reanimação em sala de parto. Não foi constatado nenhum óbito ou doença entre os recém-nascidos do grupo controle.

Os recém-nascidos de muito baixo peso (casos) apresentaram uma média de peso ao nascer de $1.114 \mathrm{~g}(\mathrm{DP}=274 \mathrm{~g})$ e uma idade gestacional média de 30,4 semanas ( $\mathrm{DP}=2,89$ ).

Utilizando-se as curvas de Alexander et al. 5, observou-se que $54 \%$ dos recém-nascidos de muito baixo peso foram classificados como adequados para a idade gestacional e $45 \%$ destes recém-nascidos foram classificados como pequenos para a idade gestacional. Não houve diferença entre os recém-nascidos adequados para idade gestacional e pequenos para idade gestacional em relação à taxa de óbito $(p=0,2)$.

O tipo de parto predominante foi o normal (51\%). Em relação às condições de nascimento, pode-se observar que $33 \%$ dos recém-nascidos de muito baixo peso nasceram com um Apgar no 1o minuto abaixo de quatro e $55,6 \%$ necessitaram de algum tipo de reanimação em sala de parto, além do oxigênio inalatório.
As Tabelas 1 e 2 contêm os dados sobre a mortalidade desses recém-nascidos estratificados pelo peso de nascimento e idade gestacional. Nessas tabelas observa-se que a mortalidade ainda é muito alta entre os recém-nascidos com peso menor do que $800 \mathrm{~g}$ e idade gestacional abaixo de 28 semanas de gestação. O limite da viabilidade dos recém-nascidos do estudo foi de $600 \mathrm{~g}$ e 26 semanas de idade gestacional. A mortalidade dos recém-nascidos com peso menor do que $1.000 \mathrm{~g}$ foi de $68,8 \%$ e dos recém-nascidos com $1.000 \mathrm{~g}$ ou mais foi de $16,5 \%$.

O tempo médio da internação dos recémnascidos de muito baixo peso que faleceram foi de 11,6 dias e dos recém-nascidos de muito baixo peso que sobreviveram foi de 49,3 dias.
Tabela 1

Número total de recém-nascidos de muito baixo peso, número e porcentagem de óbitos segundo o peso de nascimento. Hospital Geral de Caxias do Sul, Rio Grande do Sul, Brasil, 1998-2004.

\begin{tabular}{lccc}
\hline $\begin{array}{l}\text { Peso de nascimento } \\
\text { (gramas) }\end{array}$ & N total & $\mathbf{n}$ & Óbitos \\
\hline $500-599$ & 7 & 7 & 100,0 \\
$600-699$ & 12 & 11 & 91,6 \\
$700-799$ & 17 & 14 & 82,3 \\
$800-899$ & 14 & 5 & 35,7 \\
$900-999$ & 11 & 5 & 45,4 \\
$1.000-1.099$ & 29 & 11 & 37,9 \\
$1.100-1.199$ & 18 & 6 & 33,3 \\
$1.200-1.299$ & 27 & 3 & 11,1 \\
$1.300-1.399$ & 26 & 2 & 7,7 \\
$1.400-1.499$ & 39 & 1 & 2,6 \\
Total & 200 & 65 & 32,5 \\
\hline
\end{tabular}

Tabela 2

Número total de recém-nascidos de muito baixo peso, número e porcentagem de óbitos segundo a idade gestacional. Hospital Geral de Caxias do Sul, Rio Grande do Sul, Brasil, 1998-2004.

\begin{tabular}{lccc}
\hline $\begin{array}{l}\text { Idade gestacional } \\
\text { (semanas) }\end{array}$ & $\mathbf{N}$ total & $\mathbf{n}$ & Óbitos \\
\hline$<28$ & 30 & 22 & $\%$ \\
$28-31$ & 90 & 31 & 73,3 \\
$32-36$ & 75 & 12 & 34,4 \\
$37-41$ & 5 & 0 & 16,0 \\
Total & 200 & 65 & 0,0 \\
\hline
\end{tabular}


A principal causa básica de óbito dos recémnascidos de muito baixo peso foi a hipertensão materna (35,3\%) e como causa imediata de óbito predominou a infecção (52,3\%).

Observou-se na análise univariada e multivariada que a média de idade das mães dos casos foi de 26,3 anos (DP = 7,41) e das mães dos controles de 25,1 (DP = 6,43), diferença sem significância estatística $(p=0,05)$. A Tabela 3 apresenta a distribuição das mães segundo as variáveis maternas.

As mães adolescentes, quando comparadas com as de 20 anos ou mais, não se constituíram em risco para o nascimento de recém-nascido de muito baixo peso. A freqüência de mães adolescentes no grupo dos casos foi de $23 \%$ e no grupo dos controles foi de 20,5\% ( $p=0,5)$. Entretanto, as gestantes com 35 anos ou mais apresentaram associação com o nascimento de recém-nascido de muito baixo peso, que persistiu após a análise multivariada.

Em relação ao grau de instrução das mães verificou-se que mais de $60 \%$ das gestantes nos dois grupos possuíam apenas o primeiro grau incompleto. Apesar de haver uma freqüência três vezes maior de mães analfabetas no grupo de casos em comparação com os controles, esta diferença não foi significante. Após a análise multivariada para controle dos fatores de confusão resultaram como fatores de risco independentes para o nascimento de recém-nascido de muito baixo peso neste grupo de variáveis a idade materna acima de 35 anos e a história de um filho anterior com baixo peso de nascimento.

Na Tabela 4 visualiza-se a distribuição dos nascidos vivos segundo variáveis da gestação. As mães dos recém-nascidos de muito baixo peso

Tabela 3

Distribuição das mães segundo variáveis maternas. Hospital Geral de Caxias do Sul, Rio Grande do Sul, Brasil, $1998-2004$.

\begin{tabular}{|c|c|c|c|c|c|c|}
\hline \multirow[t]{2}{*}{ Variáveis maternas } & \multicolumn{2}{|c|}{ Casos * $(n=200)$} & \multicolumn{2}{|c|}{ Controles * $(n=400)$} & \multirow{2}{*}{$\begin{array}{l}\text { OR bruta } \\
\text { (IC95\%) }\end{array}$} & \multirow{2}{*}{$\begin{array}{l}\text { OR ajustada } \\
\text { (IC95\%) }\end{array}$} \\
\hline & $\mathrm{n}$ & $\%$ & $\mathrm{n}$ & $\%$ & & \\
\hline \multicolumn{7}{|l|}{ Idade (anos) } \\
\hline$\geq 35$ & 38 & 19,0 & 34 & 8,5 & $2,52(1,53-4,15)$ & $2,57(1,23-5,38)$ \\
\hline$<35 \star \star$ & 162 & 81,0 & 366 & 91,5 & 1,00 & 1,00 \\
\hline \multicolumn{7}{|l|}{ Analfabetismo } \\
\hline Sim & 6 & 3,0 & 3 & 0,8 & $4,08(1,01-16,50)$ & $3,57(0,72-17,53)$ \\
\hline Não ** & 192 & 97,0 & 392 & 99,2 & 1,00 & 1,00 \\
\hline \multicolumn{7}{|l|}{ Situação conjugal } \\
\hline Sem companheiro & 45 & 23,0 & 53 & 13,3 & $1,94(1,25-3,02)$ & $0,82(0,24-2,70)$ \\
\hline Com companheiro ** & 151 & 77,0 & 346 & 86,7 & 1,00 & 1,00 \\
\hline \multicolumn{7}{|c|}{ Renda per capita (salário mínimo) } \\
\hline$<0,5$ & 39 & 24,5 & 51 & 15,9 & $1,72(1,07-2,75)$ & $1,88(0,85-4,14)$ \\
\hline$\geq 0,5$ ** & 120 & 75,5 & 270 & 84,1 & 1,00 & 1,00 \\
\hline \multicolumn{7}{|l|}{ Gestações } \\
\hline $1-3 * \star$ & 150 & 75,4 & 319 & 79,8 & 1,00 & 1,00 \\
\hline 4 e mais & 49 & 24,6 & 81 & 20,2 & $1,28(0,85-1,92)$ & $0,53(0,25-1,09)$ \\
\hline \multicolumn{7}{|l|}{ Abortos } \\
\hline Sim & 41 & 20,6 & 49 & 12,2 & $1,85(1,17-2,93)$ & $2,27(0,89-5,78)$ \\
\hline Não ** & 158 & 79,4 & 351 & 87,8 & 1,00 & 1,00 \\
\hline \multicolumn{7}{|l|}{ Natimortos } \\
\hline Sim & 8 & 4,0 & 4 & 1,0 & $4,16(1,24-14,01)$ & $3,27(0,56-19,15)$ \\
\hline Não ** & 190 & 96,0 & 396 & 99,0 & 1,00 & 1,00 \\
\hline \multicolumn{7}{|l|}{ Prematuros } \\
\hline Sim & 26 & 13,1 & 22 & 5,5 & $2,59(1,43-4,71)$ & $0,63(0,18-2,14)$ \\
\hline Não ** & 172 & 86,9 & 378 & 94,5 & 1,00 & 1,00 \\
\hline \multicolumn{7}{|l|}{ Baixo peso anterior } \\
\hline Sim & 49 & 43,4 & 36 & 14,5 & $4,53(2,71-7,56)$ & $4,38(2,25-8,53)$ \\
\hline Não ** & 64 & 56,6 & 213 & 85,5 & 1,00 & 1,00 \\
\hline
\end{tabular}

* As diferenças são decorrentes de dados sem informação ou perdidos;

** Referência. 
Distribuição dos nascidos vivos segundo variáveis da gestação. Hospital Geral de Caxias do Sul, Rio Grande do Sul, Brasil, $1998-2004$.

\begin{tabular}{|c|c|c|c|c|c|c|}
\hline \multirow[t]{2}{*}{ Variáveis } & \multicolumn{2}{|c|}{ Casos * $(n=200)$} & \multicolumn{2}{|c|}{ Controles * $(n=400)$} & \multirow{2}{*}{$\begin{array}{l}\text { OR bruta } \\
\text { (IC95\%) }\end{array}$} & \multirow{2}{*}{$\begin{array}{l}\text { OR ajustada } \\
\text { (IC95\%) }\end{array}$} \\
\hline & $\mathrm{n}$ & $\%$ & $\mathrm{n}$ & $\%$ & & \\
\hline \multicolumn{7}{|c|}{ Consultas pré-natais } \\
\hline Nenhuma & 36 & 18,5 & 13 & 3,3 & $6,60(3,40-12,78)$ & $16,81(6,20-45,58)$ \\
\hline 1 ou mais ** & 159 & 81,5 & 379 & 96,7 & 1,00 & 1,00 \\
\hline \multicolumn{7}{|c|}{ 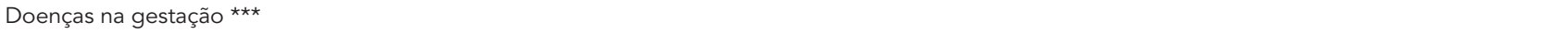 } \\
\hline Sim & 121 & 60,5 & 122 & 30,5 & $3,49(2,44-4,97)$ & $2,13(1,05-4,30)$ \\
\hline Não ** & 79 & 39,5 & 278 & 69,5 & 1,00 & 1,00 \\
\hline \multicolumn{7}{|c|}{ Hipertensão materna } \\
\hline Sim & 70 & 35,0 & 27 & 6,8 & $7,43(4,57-12,10)$ & $3,49(1,41-8,63)$ \\
\hline Não ** & 130 & 65,0 & 373 & 93,3 & 1,00 & 1,00 \\
\hline \multicolumn{7}{|l|}{ Diabete } \\
\hline Sim & 8 & 4,0 & 5 & 1,3 & $3,29(1,06-10,19)$ & $1,65(0,31-8,71)$ \\
\hline Não ** & 192 & 96,0 & 395 & 98,7 & 1,00 & 1,00 \\
\hline \multicolumn{7}{|l|}{ Corioamnionite } \\
\hline Sim & 19 & 9,5 & 1 & 0,3 & $41,88(5,56-315,2)$ & 1,00 \\
\hline Não ** & 181 & 90,5 & 399 & 99,7 & 1,00 & 1,00 \\
\hline \multicolumn{7}{|l|}{ Sífilis, Toxo e HIV } \\
\hline Sim & 13 & 6,5 & 16 & 4,0 & $1,66(0,78-3,54)$ & $0,18(0,01-3,10)$ \\
\hline Não ** & 187 & 93,5 & 384 & 96,0 & 1,00 & 1,00 \\
\hline \multicolumn{7}{|c|}{ Internação materna } \\
\hline Sim & 68 & 34,5 & 12 & 3,0 & $17,04(8,94-32,49)$ & $12,37(4,79-31,90)$ \\
\hline Não ** & 129 & 65,5 & 388 & 97,0 & 1,00 & 1,00 \\
\hline \multicolumn{7}{|l|}{ Ruprema \# > 24h } \\
\hline Sim & 32 & 16,0 & 2 & 0,5 & $37,90(8,98-159,9)$ & 1,00 \\
\hline Não ** & 168 & 84,4 & 398 & 99,5 & 1,00 & 1,00 \\
\hline \multicolumn{7}{|c|}{ Drogas na gestação } \\
\hline Sim & 11 & 5,5 & 9 & 2,3 & $2,52(1,03-6,20)$ & $2,60(0,28-23,45)$ \\
\hline Não ** & 189 & 94,5 & 391 & 97,8 & 1,00 & 1,00 \\
\hline \multicolumn{7}{|c|}{ Fumo na gestação } \\
\hline Sim & 40 & 20,1 & 56 & 14,4 & $1,54(0,98-2,41)$ & $0,90(0,36-2,28)$ \\
\hline Não ** & 159 & 79,9 & 344 & 86,0 & 1,00 & 1,00 \\
\hline
\end{tabular}

* As diferenças são decorrentes de dados sem informação ou perdidos;

** Referência;

*** Inclui qualquer doença apresentada pela gestante durante a gestação;

\# Ruptura precoce de membranas.

realizaram em média quatro consultas durante a gestação, enquanto as gestantes dos recém-nascidos do grupo controle fizeram 6,5 consultas em média $(\mathrm{p}<0,001)$. Houve um grande número de gestantes sem nenhuma consulta de pré-natal no grupo dos recém-nascidos de muito baixo peso $(18,5 \%)$. A hipertensão (35\%) e as doenças infecciosas (16\%) foram as principais doenças maternas no grupo dos recém-nascidos com muito baixo peso.

Na Tabela 4 pode-se observar, ainda, que, embora a maioria das variáveis apresentadas estivesse associada ao nascimento de recém-nas- cido de muito baixo peso, após a análise multivariada, persistiram como fatores de risco para o nascimento de recém-nascido de muito baixo peso somente a ausência de pré-natal, a internação materna durante a gestação, a presença de doenças maternas na gestação e a hipertensão materna, sendo que a ausência de pré-natal e a internação materna na gestação foram as variáveis que apresentaram a maior magnitude de associação com o nascimento de recém-nascido de muito baixo peso. 


\section{Discussão}

A associação entre idade materna avançada e nascimento prematuro foi observada no presente estudo, corroborando os achados de outros autores como Tough et al. 6 , que estudando o impacto da idade materna acima de 35 anos sobre as gestações, verificaram um aumento nos nascimentos nesta faixa etária de 51\% entre 1990 e 1996. Esses nascimentos foram responsáveis por um crescimento de $43 \%$ na taxa de partos prematuros, sendo este aumento devido, basicamente, às complicações obstétricas relacionadas à idade materna.

Para a análise do nível sócio-econômico optou-se por classificar a situação econômica da família segundo a renda per capita, não se constatando associação entre rendimento e o nascimento de recém-nascido de muito baixo peso. Esse fato, provavelmente, ocorreu devido às características da população do estudo, com grande predomínio de gestantes de baixo nível sócioeconômico e educacional. Vale lembrar que os dados são secundários podendo apresentar viés de informação.

Collins et al. 7 mostraram, em seu estudo, que a disparidade de renda entre as mulheres americanas negras e brancas tem sido um dos fatores responsáveis pelo aumento das taxas de recémnascidos de muito baixo peso.

A maior parte das mães possuía apenas o primeiro grau incompleto. Entretanto, observou-se uma freqüência três vezes maior de mães analfabetas no grupo de casos do que no de controles. Esse achado não apresentou significância estatística, provavelmente pelo pequeno número de gestantes analfabetas.

Evans \& Rosen ${ }^{8}$ referem que mulheres sem união estável têm taxas mais altas de nascimentos prematuros do que as com união estável, fato este não encontrado no presente estudo.

A história das gestações anteriores em relação a abortos, natimortos e prematuros freqüentemente está relacionada com o nascimento de recém-nascidos prematuros e de muito baixo peso. Gardosi et al. ${ }^{9}$ observaram que a história anterior de parto prematuro foi o mais forte fator de risco para o nascimento antes de 34 semanas de idade gestacional. Eles concluíram que a repetição do parto prematuro pode ser, pelo menos parcialmente, devido a um componente genético.

No estudo foi encontrada a associação da história de um recém-nascido anterior com baixo peso ao nascer com o nascimento de recém-nascido de muito baixo peso.

Vintzileos et al. 10, estudando a associação entre pré-natal e nascimentos prematuros nos Estados Unidos, entre 1995 e 1998, verificaram que a taxa de prematuros foi de $9,2 \%$ quando as gestantes faziam pelo menos uma consulta de pré-natal e de $25,6 \%$ quando não freqüentavam o pré-natal. Eles verificaram que a influência do pré-natal no nascimento prematuro manifestou-se mesmo na presença de condições clínicas adversas, como hipertensão, diabete e doença renal.

No estudo, a média de consultas de pré-natal das mães dos recém-nascidos de muito baixo peso foi de quatro e no grupo controle de seis. Essa diferença deve ser analisada com cuidado porque as gestantes do grupo caso tiveram menor tempo disponível para a realização do prénatal devido ao parto prematuro que, em geral, ocorreu antes das 32 semanas de gestação, sendo que o número menor de consultas pode não ser a causa do nascimento prematuro e sim a conseqüência. Ao analisar o número de gestantes que não fizeram nenhuma consulta de pré-natal, observou-se que $18,5 \%$ das mães dos recém-nascidos de muito baixo peso estavam nesta situação em comparação com apenas 3,3\% das mães do grupo controle. Essa diferença foi estatisticamente significante mostrando que a ausência de prénatal está fortemente associada ao nascimento de recém-nascidos de muito baixo peso.

Kilsztajn et al. 11, em São Paulo, comprovaram que quanto maior o número de consultas médicas no pré-natal, menor a prevalência de recém-nascidos de baixo peso e prematuros. Adicionalmente, houve uma redução da diferença na prevalência de baixo peso ao nascer e prematuridade de $14 \%$ para $4 \%$ com o aumento das visitas de zero a três para sete ou mais durante a gestação.

Aragão et al. 12, em São Luís do Maranhão, realizaram um estudo com o objetivo de identificar os fatores de risco para prematuridade. A incidência de prematuridade foi de $12,7 \%$ e os fatores de risco, após a análise múltipla, foram a idade materna menor do que 18 anos e a ausência de pré-natal.

Analisou-se a associação do uso de drogas e o hábito de fumar durante a gestação com o nascimento de recém-nascido de muito baixo peso. Em relação ao fumo encontrou-se $20 \%$ de fumantes entre as mães dos recém-nascidos de muito baixo peso e $14 \%$ entre as mães do grupo controle, diferença esta sem significância estatística. Quanto à utilização de outras drogas, predominou o uso de crack e álcool, sendo a sua freqüência de 3,3\%. A prevalência do uso dessas drogas foi obtida usando-se como único método de pesquisa a entrevista com a mãe e, portanto, é provável que esse número seja mais elevado. Cunha et al. 13 encontraram uma prevalência de $4,6 \%$ de gestantes expostas à cocaína, utilizando 
como métodos de investigação a entrevista com as mães e a pesquisa de drogas no mecônio.

Embora se observe relatos na literatura sobre o aumento dos partos prematuros relacionado com o abuso de drogas 14, não foi encontrado aumento no risco de nascimentos de recém-nascido de muito baixo peso associado ao abuso destas substâncias pela gestante, provavelmente, pelo pequeno número de gestantes envolvidas.

Em relação às doenças na gestação, a hipertensão materna foi a principal causa clínica do desencadeamento do parto prematuro, apresentando associação estatisticamente significante com o nascimento de recém-nascido de muito baixo peso. Esses achados são descritos na literatura médica e a causa provável é a má perfusão placentária originada pela vasoconstrição e pelo deficiente crescimento placentário ${ }^{15}$. A hipertensão materna certamente contribuiu para o grande número de recém-nascidos pequenos para a idade gestacional encontrado no grupo dos casos.

Como segunda doença mais freqüente na gestação das mães dos recém-nascidos de muito baixo peso encontrou-se as infecções. Chamou a atenção o grande número de mães com sífilis, toxoplasmose e AIDS em ambos os grupos.

A relação da infecção/inflamação com a prematuridade é bem conhecida. Em estudo perinatal colaborativo 16 , a corioamnionite esteve presente em $6 \%$ de 43.940 partos. A freqüência aumentou com a diminuição da idade gestacional: $15 \%$ entre $28-32$ semanas, $8 \%$ entre $33-36$ e $5 \%$ após as 36 semanas de gestação.

A bacteriúria assintomática tem sido associada com a prematuridade há muitos anos. A revisão sistemática conduzida por Villar et al. 17 concluiu que o tratamento da bacteriúria assintomática diminui a ocorrência de prematuridade e o nascimento de recém-nascidos de muito baixo peso.

Faro 18 mostrou que os microorganismos associados com o nascimento pré-termo são capazes de produzir prostaglandinas diretamente ou através da produção de fosfolipase A2, a qual libera ácido aracdônico resultando em aumento das prostaglandinas.

Assim, alguns autores como Hauth et al. 19, têm recomendado o uso de antibióticos na prevenção do parto prematuro para tratar a infecção gênito-urinária, prevenir a ruptura precoce de membranas, reduzir o risco de infecção após a ruptura da bolsa e tratar do trabalho de parto prematuro espontâneo de etiologia infecciosa.

As doenças infecciosas em conjunto com a doença hipertensiva contribuíram, no estudo, com mais de $70 \%$ das doenças da gestação. Essas podem ser evitadas ou ter os seus efeitos mini- mizados por meio de uma maior freqüência ao pré-natal e de acompanhamento qualificado à gestante de risco.

No presente estudo, o grupo de gestantes de risco foi constituído por gestantes sem pré-natal, com idade igual ou maior do que 35 anos, que foram internadas durante a gestação ou apresentaram doenças na gestação, principalmente, a hipertensão e as infecções gênito-urinárias, e que tinham história prévia de um filho anterior pesando menos do que $2.500 \mathrm{~g}$.

Resultados semelhantes foram observados por Spallicci et al. 20 que encontraram a hipertensão arterial materna, o número de consultas realizadas no pré-natal, a prematuridade anterior e ruptura precoce das membranas como variáveis que se associaram à prematuridade.

Estudo realizado na Alemanha 21, em 1994, verificou como fatores de risco para o nascimento prematuro a história de aborto, natimorto ou prematuro prévio, a idade materna acima de 35 anos e abaixo de 18, a presença de pré-eclâmpsia e corioamnionite.

Alguns autores têm demonstrado que mulheres que sofrem discriminação racial ao longo da vida apresentam um risco aumentado para o nascimento de recém-nascido de muito baixo peso mostrando, assim, a complexidade e multiplicidade dos fatores envolvidos no nascimento prematuro 22.

A maioria das gestantes do estudo iniciou as consultas de pré-natal tardiamente, no segundo trimestre de gravidez. Esse fato, aliado à dificuldade do SUS de proporcionar exames na rapidez necessária e um atendimento qualificado, tem gerado muitos nascimentos de recém-nascidos de muito baixo peso causados por doenças evitáveis, diferentemente dos países desenvolvidos, onde os nascimentos prematuros têm aumentado devido a um maior número de gestações múltiplas.

Os esforços para diminuir os partos prematuros e o nascimento de recém-nascidos de muito baixo peso devem ser realizados na atenção prénatal, que precisa ser iniciada no primeiro trimestre da gestação e ser de qualidade. É fundamental que as gestantes com patologias de risco ou que possuam fatores associados ao nascimento prematuro na sua história prévia sejam identificadas precocemente e encaminhadas para ambulatórios de gestantes de risco, onde possam receber atendimento personalizado e voltado às suas necessidades médicas e sociais.

Diaz et al. 23 relataram diminuição na freqüência de recém-nascidos de muito baixo peso para $0,85 \%$ do total de nascimentos por meio de programas de baixo custo, que têm como alicerce a atenção pré-natal qualificada. Essa valorização 
do atendimento pré-natal gerou uma importante redução na mortalidade perinatal, que caiu de 16,7 por mil nascidos vivos em 1988 , para 7,2 por mil nascidos vivos em 1996.

É importante enfatizar que o custo econômico para se evitar o nascimento de recém-nascidos de muito baixo peso é muito menor do que o custo do tratamento destes em unidades de tratamento intensivo.

\section{Resumo}

Identificar os fatores de risco associados ao nascimento de recém-nascidos de muito baixo peso no Hospital Geral de Caxias do Sul, Rio Grande do Sul, Brasil. Estudo de caso-controle, abrangendo 200 recém-nascidos com peso entre 500 e $1.499 \mathrm{~g}$ (casos) e 400 recém-nascidos com peso entre 3.000 e $3.999 \mathrm{~g}$ (controles). Incluídos recém-nascidos de gestação única, cujas mães não realizaram pré-natal ou o fizeram na rede pública. A variável dependente foi o peso de nascimento e as variáveis independentes foram as sócio-econômicas, educacionais, gestacionais e do parto. Utilizou-se a análise univariada e multivariada, com nível de significância de 5\%. A mortalidade dos recém-nascidos de muito baixo peso foi de $32,5 \%$. O limite de viabilidade dos recém-nascidos foi $600 \mathrm{~g}$ de peso e 26 semanas de idade gestacional. As variáveis relacionadas com o nascimento prematuro foram: idade materna $\geq 35$ anos ( $p=$ $0,01)$, ausência de pré-natal ( $p<0,0001)$, doenças na gestação $(p=0,03)$, hipertensão materna ( $p=0,007)$, internação na gestação $(p<0,0001)$ e filho anterior de baixo peso ao nascer $(p<0,0001)$. Muitos nascimentos prematuros ocorreram por causas evitáveis.

Recém-Nascido de Baixo Peso; Prematuro; Pobreza
Concluindo, é por meio de programas preventivos e de baixo custo, elaborados com base em estudos epidemiológicos, que se pode diminuir o nascimento de recém-nascidos de muito baixo peso e, conseqüentemente, influenciar positivamente a redução das taxas de mortalidade infantil.

\section{Colaboradores}

B. F. Araújo participou da elaboração do estudo, coleta e análise dos dados, pesquisa bibliográfica e redação do artigo. A. C. d'A. Tanaka participou da elaboração do estudo, análise dos dados e redação final do artigo. 


\section{Referências}

1. Barreto ML, Carmo EH. Mudanças em padrões de morbimortalidade: conceitos e métodos. In: Monteiro CA, organizador. Velhos e novos males da saúde no Brasil. A evolução do país e de suas doenças. São Paulo: Editora Hucitec/Universidade de São Paulo; 1995. p. 17-30.

2. Roth J, Resnick MB, Ariet M, Carter RL, Eitzman DV, Curran JS, et al. Changes in survival patterns of very low-birth-weight infants from 1980 to 1993. Arch Pediatr Adolesc Med 1995; 149:1311-7.

3. Alexander GR, Slay M. Prematurity at birth: trends, racial disparities, and epidemiology. Ment Retard Dev Disabil Res Rev 2002; 8:215-20.

4. Kramer MS, Goulet L, Lydon J, Seguin L, McNamara H, Dassa C, et al. Socio-economic disparities in preterm birth: causal pathways and mechanisms. Paediatr Perinat Epidemiol 2001; 15 Suppl 2:10423.

5. Alexander GR, Himes JH, Kaufman RB, Mor J, Kogan M. A United States national reference for fetal growth. Obstet Gynecol 1996; 87:163-8.

6. Tough SC, Newburn-Cook C, Johnston DW, Svenson LW, Rose S, Belik J. Delayed childbearing and its impact on population rate changes in lower birth weight, multiple birth, and preterm delivery. Pediatrics 2002; 109:399-403.

7. Collins Jr. JW, Herman AA, David RJ. Very-lowbirthweight infants and income incongruity among African American and white parents in Chicago. Am J Public Health 1997; 87:414-7.

8. Evans MA, Rosen LN. Demographic and psychosocial risk factors for preterm delivery in an active duty pregnant population. Mil Med 2000; 165:4953.

9. Gardosi J, Francis A. Early pregnancy predictors of preterm birth: the role of a prolonged menstruation-conception interval. BJOG 2000; 107:228-37.

10. Vintzileos AM, Ananth CV, Smulian JC, Scorza WE, Knuppel RA. The impact of prenatal care in the United States on preterm births in the presence and absence of antenatal high-risk conditions. Am J Obstet Gynecol 2002; 187:1254-7.

11. Kilsztajn S, Rossbach A, Carmo MS, Sugahara GT. Assistência pré-natal, baixo peso e prematuridade no Estado de São Paulo, 2000. Rev Saúde Pública 2003; 37:303-10.

12. Aragão VM, Silva AA, Aragão LF, Barbieri MA, Bettiol H, Coimbra LC, et al. Risk factors for preterm births in São Luís, Maranhão, Brazil. Cad Saúde Pública 2004; 20:57-63.
13. Cunha GB, Rotta NT, Silva AR, Dieder AL, Wolf AL, Moser C, et al. Prevalence of prenatal exposure to cocaine in a sample of newborns from a university teaching hospital. J Pediatr (Rio J) 2001; 77:369-73.

14. Spence MR, Williams R, DiGregorio GJ, Kirby-McDonnell A, Polansky M. The relationship between recent cocaine use and pregnancy outcome. $\mathrm{Ob}$ stet Gynecol 1991; 78(3 Pt 1):326-9.

15. Kim CR, Vohr BR, Oh W. Effects of maternal hypertension in very-low-birth-weight infants. Arch Pediatr Adolesc Med 1996; 150:686-91.

16. Williams MC, O'Brien WF, Nelson RN, SpellacyWN. Histologic chorioamnionitis is associated with fetal growth restriction in term and preterm infants. Am J Obstet Gynecol 2000; 183:1094-9.

17. Villar J, Gulmezoglu AM, de Onis M. Nutritional and antimicrobial interventions to prevent preterm birth: an overview of randomized controlled trials. Obstet Gynecol Surv 1998; 53:575-85.

18. Faro S. Screening for bacterial vaginosis. Infect Dis Obstet Gynecol 1998; 6:235.

19. Hauth JC, Goldenberg RL, Andrews WW, DuBard MB, Copper RL. Reduced incidence of preterm delivery with metronidazole and erythromycin in women with bacterial vaginosis. N Engl J Med 1995; 333:1732-6.

20. Spallicci MDB, Chiea MA, Albuquerque PB, Bittar RE, Zugaib M. Estudo de algumas variáveis maternas relacionadas com a prematuridade no Hospital Universitário da Universidade de São Paulo. Rev Med Hosp Univ 2000; 10:19-23.

21. Martius JA, Steck T, Oehler MK, Wulf KH. Risk factors associated with preterm $(<37+0$ weeks $)$ and early preterm birth $(<32+0$ weeks): univariate and multivariate analysis of 106345 singleton births from the 1994 statewide perinatal survey of Bavaria. Eur J Obstet Gynecol Reprod Biol 1998; 80:1839.

22. Collins Jr. JW, David RJ, Handler A, Wall S, Andes S. Very low birthweight in African American infants: the role of maternal exposure to interpersonal racial discrimination. Am J Public Health 2004; 94:2132-8.

23. Díaz-Alvarez M, Bello-Machado P, Portuondo $\mathrm{M}$, Odelliín G, Medina-Garcia M. Incidencia, morbilidad y mortalidad neonatal en recién nacidos de menos de 1500 gramos de peso. Rev Cuba Pediatr 1993; 65:81-7.

Recebido em 16/Out/2006

Versão final reapresentada em 25/Abr/2007

Aprovado em 16/Mai/2007 